\title{
Histopathological evidence of invasive gastric mucormycosis after transarterial chemoembolization and liver transplantation
}

\author{
P. Kaiser - E. M. Maggio - T. Pfammatter - B. Misselwitz $\cdot$ \\ S. Flury $\cdot$ P. M. Schneider $\cdot$ P. Dutkowski $\cdot$ S. Breitenstein $\cdot$ \\ B. Müllhaupt $\cdot$ P. A. Clavien $\cdot$ N. J. Mueller
}

Received: 6 December 2013/Accepted: 15 February 2014/Published online: 5 March 2014

(C) Springer-Verlag Berlin Heidelberg 2014

\begin{abstract}
We describe a case of a 62-year-old diabetic woman with hepatocellular carcinoma due to chronic hepatitis B virus infection. Two weeks after orthotopic liver transplantation, endoscopy for massive upper gastrointestinal bleeding revealed a large necrotic area in the gastric fundus. The patient underwent emergency resection. Histopathologically, angioinvasive mold infection compatible with mucormycosis was diagnosed in a large area of necrosis, mimicking an atypically localized gastric ulcer. Foreign bodies originating from transarterial chemoembolization (TACE) performed 7 and 8 months earlier and 40 days before transplantation were identified in the submucosal tissue. The patient
\end{abstract}

P. Kaiser · N. J. Mueller $(\bowtie)$

Division of Infectious Diseases and Hospital Epidemiology, Department of Medicine, University Hospital Zürich,

Rämistrasse 100/RAE U 70, 8091 Zürich, Switzerland

e-mail: nicolas.mueller@usz.ch

E. M. Maggio

Institute of Clinical Pathology, University Hospital Zürich,

Zürich, Switzerland

T. Pfammatter

Institute of Diagnostic and Interventional Radiology,

University Hospital Zürich, Zürich, Switzerland

B. Misselwitz · B. Müllhaupt

Division of Gastroenterology, University Hospital Zürich,

Zürich, Switzerland

\section{S. Flury}

Division of Nephrology, University Hospital Zürich, Zürich, Switzerland

P. M. Schneider · P. Dutkowski - S. Breitenstein · P. A. Clavien Division of Transplantation and Visceral Surgery,

University Hospital Zürich, Zürich, Switzerland was treated with liposomal amphotericin B (LAB) for 5 weeks, followed by 7 weeks of posaconazole. Followup biopsies after 1 and 5 months confirmed successful treatment. Review of the radiological images of the TACE procedure showed that some of the TACE material had been diverted to the stomach via an accessory gastric branch originating from the left hepatic artery. TACE agents may be associated with chronic, refractory gastroduodenal ulcers. We hypothesize that the ischemic lesion was first colonized with presumed Mucorales mold and invasive growth was promoted by the posttransplantation immunosuppression. Careful exploration of extrahepatic collaterals during TACE may prevent this complication.

\section{Introduction}

The term "mucormycosis" denotes angioinvasive infection by a few species of the fungal order of Mucorales. The high-level taxonomy is currently evolving and the number of recognized pathogenic species is increasing [1]. Most isolates belong to the genera of Rhizopus, Mucor, Rhizomucor, Lichtheimia, Apophysomyces, and Cunninghamella [2]. The agents of mucormycosis are ubiquitous molds found in decaying organic matter and soil. Infection results from the inhalation of spores or inoculation through disrupted skin or mucosa [3].

Mucorales can infect a more heterogeneous range of human hosts and organs than other molds; yet, there are specific risk factors: classically, people with poorly controlled diabetes mellitus are prone to rhino-orbito-cerebral disease, and skin breaches caused by trauma, surgery, or burns may entail local soft tissue involvement. These forms of the disease predominate in the developing world and in 
studies including older cases [2, 4]. In the last two decades, the epidemiology has appeared to shift towards more conventional risk factors, i.e., prolonged neutropenia due to hematologic malignancies and immunosuppression for solid organ transplantation (SOT), that more often entail pulmonary involvement and a higher propensity for dissemination $[2,3,5,6]$. Possible reasons for this change are evolving treatment options, more widespread prophylactic/ preemptive use of antifungals non-effective for mucormycosis, and a potential publication bias. In SOT patients, the most frequent sites of initial infection are the lungs, followed by rhino-cerebral, skin/soft tissue, and gastrointestinal involvement [7].

Here, we report a case of presumed gastric mucormycosis in an immunosuppressed patient with preexisting chronic ischemic lesions due to aberrant transarterial chemoembolization (TACE) material.

\section{Case report}

A 63-year-old patient of Southeast Asian descent was referred for the treatment of a newly diagnosed hepatocellular carcinoma arising from chronic HBe antigen-negative hepatitis $\mathrm{B}$ virus infection. Comorbidities were an insulin-dependent diabetes mellitus type 2, arterial hypertension, and grade 2 chronic kidney disease. The initial staging studies indicated widespread involvement of liver segments II through IVb and VII. Tumor down-staging and temporary control by TACE and scheduling for orthotopic liver transplantation (OLT) was planned.

Shortly after the first TACE session, the patient suffered from diffuse mucosal bleeding in the gastric corpus. Endoscopically, tumor infiltration was suspected, but histology revealed extensive non-neoplastic ischemic-ulcerative alterations, presumably due to accidental TACE spillover to an arterial branch supplying the stomach. Indeed, a retrospective analysis of deeper tissue sections revealed TACE material. Endoscopic controls confirmed complete recovery of the gastric mucosa. TACE was repeated after 1 and 6 months.

The patient received a deceased-donor organ 40 days after TACE had been repeated due to tumor progression. Mycophenolate mofetil $500 \mathrm{mg}$ bid and a taper of highdose methylprednisolone was given for the induction of immunosuppression, Tacrolimus $1 \mathrm{mg}$ bid was added after 1 week. Gastroscopy for the placement of a nasoduodenal tube again revealed non-bleeding corporal ulcerations (Forrest III).

Two weeks after OLT, the patient incurred hemorrhagic shock due to massive upper gastrointestinal bleeding. A diffuse source around the previously mentioned lesions was identified (Forrest Ib) but could not be stopped by endoscopic adrenalin injections. The patient underwent emergency ulcer resection (Fig. 1a).

Histopathological work-up showed a perforated ulcer with invasively growing mold down to the muscular layer of the stomach wall (Fig. 1b). The microscopic feature of broad (about $10 \mu \mathrm{m}$ ), randomly, and sparsely septate hyphae was consistent with mucormycosis (Fig. 1c). Moreover, adjacent submucosal tissue revealed intravascular amorphous, basophilic material arising from TACE (Fig. 1d). Panfungal polymerase chain reaction (PCR) subsequently performed from paraffin-embedded tissue resulted negative. We consider these results as being falsenegative due to the minute amounts of material available and reduced recovery of DNA from embedded tissues. Fungal cultures could not be performed retrospectively since no native tissue was left over. So, the diagnosis of mucormycosis relies entirely on the morphology studies in this case.

The pre-TACE celiac angiogram revealed a tiny left hepatic branch running off to the stomach (Fig. 2a). In the review of a reformatted coronal $\mathrm{C}$-arm computed tomography (CT) image, this accessory gastric branch could be identified with a corresponding enhancement of a circumscript area in the gastric fundus (Fig. 2b). This finding corresponding to the secondary ulcer was appreciated only retrospectively.

Thirty-six hours after ulcer resection, antifungal treatment with liposomal amphotericin B (LAB) was started ( $7.5 \mathrm{mg} / \mathrm{kg} /$ day). Treatment with mycophenolate was suspended for 3 weeks and tacrolimus was withheld for 1 week, while immunosuppression with prednisolone was maintained at a dose of $20 \mathrm{mg} /$ day. An endoscopic control after 5 weeks showed moderate chronic, focally active gastritis without persisting mold infection. After 5 weeks of full-dose LAB administration, antifungal treatment was switched to posaconazole at a dose of $800 \mathrm{mg} / \mathrm{day}$ for another 7 weeks. An additional biopsy 2 months later was confirmative of successful antifungal treatment.

The postoperative course was complicated by repeated bouts of bacterial septicemia, cytomegalovirus (CMV) reactivation, and by the need for renal replacement therapy. Acute on chronic renal failure in this patient was certainly multifactorial, but may have been aggravated by prolonged high-dose LAB administration.

Nine weeks after OLT, the patient could be sent to rehabilitation and was later discharged home. However, 5 months after transplantation, she had to be readmitted due to progressive chronic graft rejection and severe CMV reactivation after the completion of secondary CMV prophylaxis. The further course of disease was unfavorable; 6 weeks later, the patient died of liver failure and additional bacterial infectious complications while waiting for retransplantation. No autopsy was performed. 

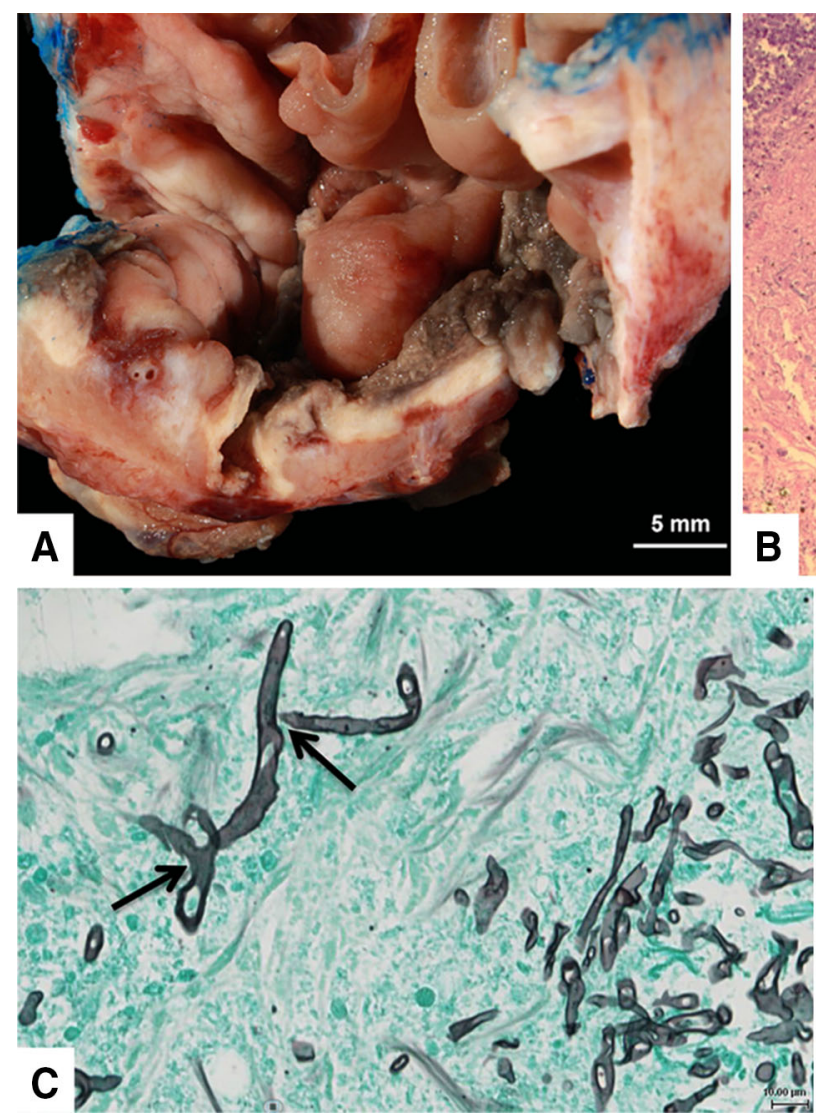
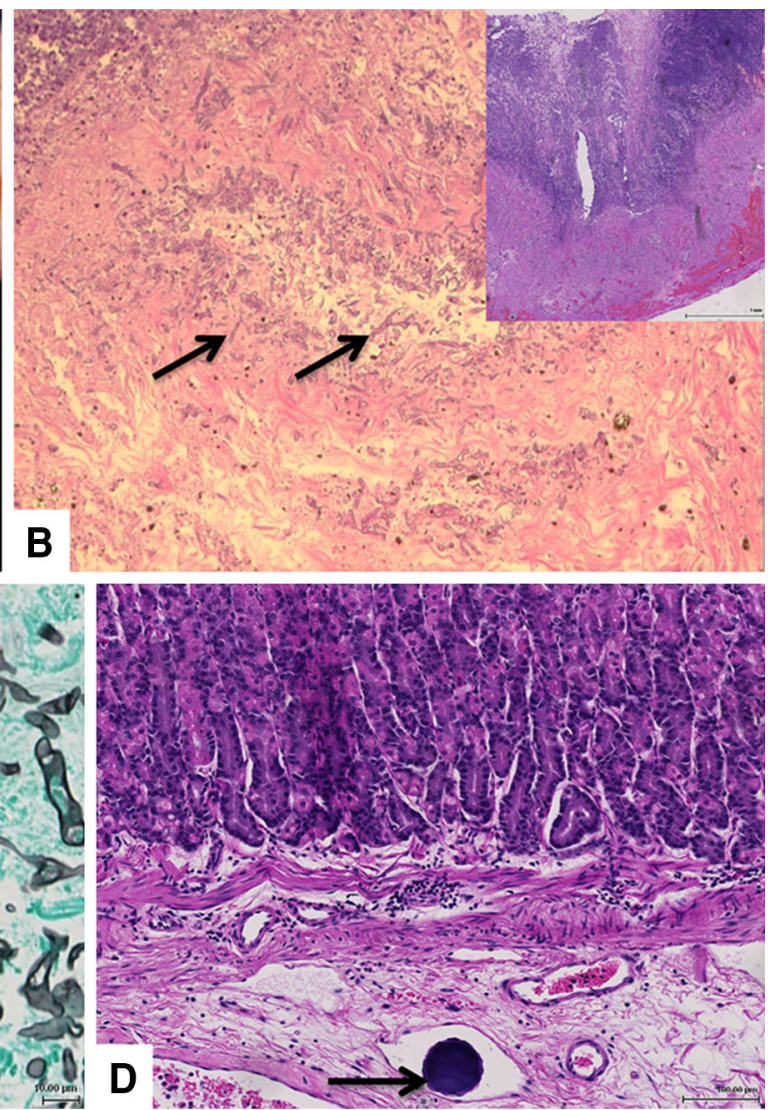

Fig. 1 Morphology of proximal gastric resection. a Gross specimen: subtotal resection with mucosal hemorrhagic necrosis and perforation. b Hematoxylin-eosin, $\times 100$ magnification: necrotic submucosal artery with invading hyphal structures (arrows) within partial mural ischemic necrosis (inlay, hematoxylin-eosin, $\times 25$ ). c Grocott's methenamine silver stain $(\mathrm{GMS}), \times 200$ : broad $(10 \mu \mathrm{m})$, ribbon-like hyphae with irregular septation and right-angle branching (arrows), typical for Mucorales molds. d Hematoxylin-eosin staining, $\times 50$ : intravascular amorphous basophilic material (arrow) arising from transarterial chemoembolization in submucosal tissue adjacent to the necrotic center of the ulcer

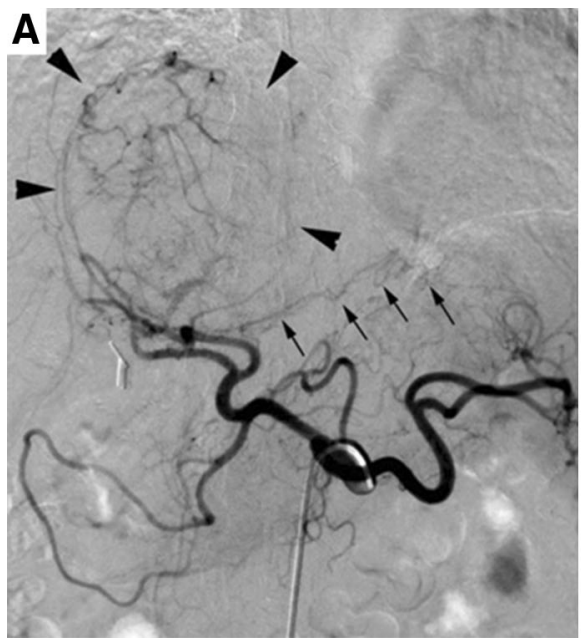

Fig. 2 Radiologic studies before the last transarterial chemoembolization (TACE). a Celiac angiogram before the last TACE. The celiac angiogram shows a large hypervascular hepatocellular carcinoma in the left liver lobe (arrowheads). The tiny accessory left hepatic artery branch (arrows) supplying the gastric fundus was overlooked, as nonselective lobar chemoembolization with $100-300 \mu \mathrm{m}$ doxorubicin-

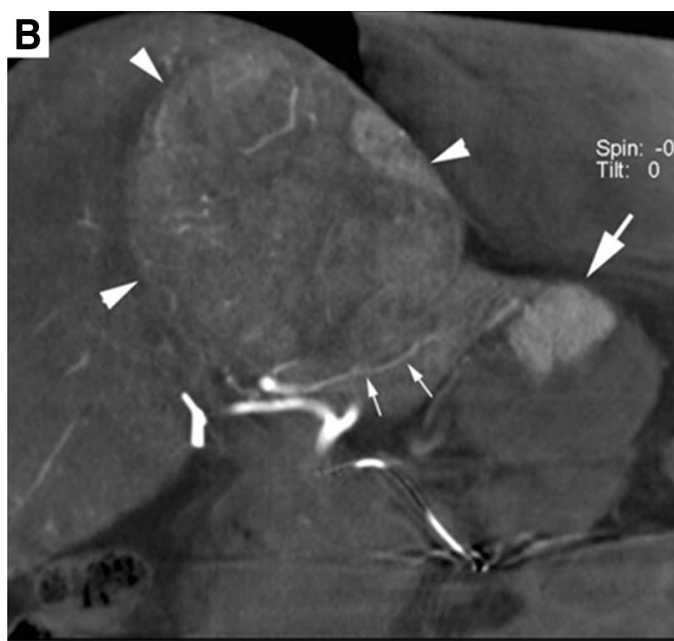

eluting particles was performed. b Computed tomography (CT) angiogram before the last TACE. The reformatted coronal C-arm CT image performed before the delivery of the particles depicts the hepatic tumor (arrowheads), the accessory gastric branch (small arrows), and enhancement of a circumscript area in the gastric fundus (large arrow) 


\section{Discussion}

Mucormycosis in SOT recipients is a rare event. According to recent TRANSNET cohort data, the cumulative 1-year incidence of invasive fungal infections is around $3 \%$. Thereof, $2 \%$ fall into the category of mucormycosis, which equates to an overall 1-year cumulative incidence of $0.07 \%$ [5, 8]. Interestingly, liver transplant recipients appear to be at a higher risk (1-year incidence $0.16 \%$ ) and suffered more often from disseminated disease than other SOT recipients $[5,7]$. This finding may indicate specific defense defects in liver transplant recipients or simply reflect a generally higher susceptibility for early fungal and bacterial infection in these often severely sick patients undergoing highly complex surgery.

In the most comprehensive case review of mucormycosis [2], gastrointestinal disease figures with $7 \%$ as a rare form with exceptionally high mortality. The literature before 1990 describes gastrointestinal mucormycosis primarily as a disease of neonates, whereas more recently, cases in patients with some underlying immunosuppression and/or peptic ulcers are reported in increasing numbers $[6$, 9]. Pathogenicity is driven by early local tissue and angioinvasion and subsequent tissue necrosis. Therefore, hemorrhage or peritonitis are often the first symptoms [6]. In our patient, expeditious surgical intervention and radical removal of the infested tissue was decisive. Indeed, the contribution of subsequent antifungal treatment to infection control remains unclear.

Our patient had several predisposing factors for invasive gastrointestinal mucormycosis; apart from long-standing (though well-controlled) diabetes mellitus and steroidbased immunosuppression, she was malnourished and suffered from renal failure. Yet, probably most crucial was a preexisting ischemic damage of the gastric mucosa due to aberrant TACE.

Non-target embolization or TACE spillover into adjacent arteries are well known to entail gastroduodenal complications in up to $9 \%$ of interventions [10]. Gastric complications mostly present as gastritis or chronic, refractory ulcers, which are rarely life-threatening [11]. No cases of fungal superinfection are reported, but the pathogenesis may be analogous to more common types of gastric ulcers. Thomson et al. [9] observed a continuum of colonization through infiltration to angioinvasion in cases of gastric mucormycosis complicating peptic ulcers. It is conceivable that, in our patient, colonization and possibly infiltration of ischemic mucosal lesions treated with acidsuppressive agents may have happened before transplantation and progressed to invasive disease in the aftermath of perioperative stress and posttransplant immunosuppression. This may explain its extraordinarily early occurrence after transplantation.
Mucormycosis remains highly lethal despite multimodal therapy. The overall mortality has declined from almost $100 \%$ to approximately $40 \%$ in the last 60 years, remaining above $75 \%$ in disseminated and gastrointestinal disease and in patients after hematopoietic stem cell transplantation [2, 7]. The cornerstones of successful management are early diagnosis, surgical debridement, improvement of the underlying conditions, and polyenebased treatment. A treatment delay of as little as 6 days has been shown to double mortality [12]. Thus, unspecific signs and symptoms may often preclude timely action. Since thrombosis of invaded vessels is a main trait of pathogenesis and precludes the efficacy of antifungal drugs with poor tissue penetration, thorough debridement of necrotic tissue is crucial and was associated with survival in many retrospective analyses [2, 7].

Besides posaconazole, only amphotericin B-based drugs have a predictable activity against mucormycosis. Experiments in mice [13] as well as, more recently, a series of non-randomized clinical studies showed the superiority of LAB and other lipid amphotericin B formulations over amphotericin B deoxycholate [7, 14]. The advantages of LAB are a better penetration into lipophilic tissues and fewer nephrotoxic effects, which may allow for sufficient dosing. Since certain agents of mucormycosis such as Cunninghamella and Rhizopus spp. display relative in vitro tolerance to the fungicidal effects of amphotericin B [15], higher dosing appears appealing. However, a randomized trial in patients with invasive mold infections (mostly Aspergillus spp.) comparing a high loading dose regimen of LAB $(10 \mathrm{mg} / \mathrm{kg})$ with lower dosing $(3 \mathrm{mg} / \mathrm{kg})$ did not show any additional benefit for higher dosing but higher rates of nephrotoxicity [16]. Whether the agents of mucormycosis behave clinically different from Aspergillus spp. in this respect remains to be determined. Meanwhile, most experts recommend $\mathrm{LAB}$ doses of at least $5 \mathrm{mg} / \mathrm{kg}$ for the treatment of mucormycosis, although few formal recommendations exist $[17,18]$.

Likewise, the question regarding a reasoned use of combination treatment remains unsettled. A matter of debate is the combination of polyenes with echinocandins, which, as monotherapeutics, have virtually no impact on Mucorales growth in vitro. Experiments in ketoacidotic mice as well as small retrospective studies suggest a synergy of caspofungin and LAB [13, 19]. Due to obvious obstacles to performing sufficiently powered randomized studies on this rare and highly heterogeneous disease, combination therapy might eventually become the de facto standard of care on the grounds of scanty evidence.

In conclusion, this report is the first-to our knowledge - to present a case of invasive infection of a preexisting gastric mucosal lesion due to aberrant TACE with mucormycosis. It demonstrates that such ischemic lesions 
may be prone to fungal infectious complications in the setting of posttransplant stress and immunosuppression. It underscores the importance of careful exploration of anatomical variant collaterals before TACE and thorough histologic work-up in unusual cases of upper gastrointestinal bleeding.

Conflict of interest All authors were directly involved in the treatment of the patient and collection ofdata, figure material, or drafting of the manuscript. All authors approved the finalversion of the paper. The authors declare that they have no conflict of interest.

\section{References}

1. Kwon-Chung KJ. Taxonomy of fungi causing mucormycosis and entomophthoramycosis (zygomycosis) and nomenclature of the disease: molecular mycologic perspectives. Clin Infect Dis. 2012;54:S8-15.

2. Roden MM, Zaoutis TE, Buchanan WL, et al. Epidemiology and outcome of zygomycosis: a review of 929 reported cases. Clin Infect Dis. 2005;41:634-53.

3. Petrikkos G, Skiada A, Lortholary O, et al. Epidemiology and clinical manifestations of mucormycosis. Clin Infect Dis. 2012;54:S23-34.

4. Chakrabarti A, Das A, Mandal J, et al. The rising trend of invasive zygomycosis in patients with uncontrolled diabetes mellitus. Med Mycol. 2006;44:335-42.

5. Park BJ, Pappas PG, Wannemuehler KA, et al. Invasive nonAspergillus mold infections in transplant recipients, United States, 2001-2006. Emerg Infect Dis. 2011;17:1855-64.

6. Spellberg B. Gastrointestinal mucormycosis: an evolving disease. Gastroenterol Hepatol (N Y). 2012;8:140-2.

7. Singh N, Aguado JM, Bonatti H, et al. Zygomycosis in solid organ transplant recipients: a prospective, matched case-control study to assess risks for disease and outcome. J Infect Dis. 2009;200:1002-11.

8. Pappas PG, Alexander BD, Andes DR, et al. Invasive fungal infections among organ transplant recipients: results of the Transplant-Associated Infection Surveillance Network (TRANSNET). Clin Infect Dis. 2010;50:1101-11.
9. Thomson SR, Bade PG, Taams M, et al. Gastrointestinal mucormycosis. Br J Surg. 1991;78:952-4.

10. Leung TK, Lee CM, Chen HC. Anatomic and technical skill factor of gastroduodenal complication in post-transarterial embolization for hepatocellular carcinoma: a retrospective study of 280 cases. World J Gastroenterol. 2005;11:1554-7.

11. Ingraham CR, Johnson GE, Nair AV, et al. Nontarget embolization complicating transarterial chemoembolization in a patient with hepatocellular carcinoma. Semin Intervent Radiol. 2011;28:202-6.

12. Chamilos G, Lewis RE, Kontoyiannis DP. Delaying amphotericin B-based frontline therapy significantly increases mortality among patients with hematologic malignancy who have zygomycosis. Clin Infect Dis. 2008;47:503-9.

13. Ibrahim AS, Avanessian V, Spellberg B, et al. Liposomal amphotericin B, and not amphotericin B deoxycholate, improves survival of diabetic mice infected with Rhizopus oryzae. Antimicrob Agents Chemother. 2003;47:3343-4.

14. Skiada A, Pagano L, Groll A, et al. Zygomycosis in Europe: analysis of 230 cases accrued by the registry of the European Confederation of Medical Mycology (ECMM) Working Group on Zygomycosis between 2005 and 2007. Clin Microbiol Infect. 2011;17:1859-67.

15. Lewis RE, Lortholary O, Spellberg B, et al. How does antifungal pharmacology differ for mucormycosis versus aspergillosis? Clin Infect Dis. 2012;54:S67-72.

16. Cornely OA, Maertens J, Bresnik M, et al. Liposomal amphotericin $\mathrm{B}$ as initial therapy for invasive mold infection: a randomized trial comparing a high-loading dose regimen with standard dosing (AmBiLoad trial). Clin Infect Dis. 2007;44: 1289-97.

17. Skiada A, Lanternier F, Groll AH, et al. Diagnosis and treatment of mucormycosis in patients with hematological malignancies: guidelines from the 3rd European Conference on Infections in Leukemia (ECIL 3). Haematologica. 2013;98:492-504.

18. Cornely OA, Arikan-Akdagli S, Dannaoui E, et al. ESCMID and ECMM Joint Clinical Guidelines for the Diagnosis and Management of Mucormycosis 2013. Clin Microbiol Infect. 2014 [Epub ahead of print].

19. Reed C, Bryant R, Ibrahim AS, et al. Combination polyene-caspofungin treatment of rhino-orbital-cerebral mucormycosis. Clin Infect Dis. 2008;47:364-71. 